\title{
Corona-Prävention im Straf- und Massnahmenvollzug
}

\section{Temporäre Einschränkungen der Therapiefrequenz und Grenzen der ausserordentlichen bedingten Entlassung}

Thierry Urwyler / Thomas Noll / Astrid Rossegger *

Die Corona-Pandemie erfordert diverse Anpassungen im Justizvollzug. Dabei sind teilweise neue, unbekannte Wege einzuschlagen. Die Autoren gehen im nachfolgenden Beitrag den Fragen nach, ob temporäre Einschränkungen der Therapiefrequenz juristisch zulässig sind und ob der anzustrebenden Reduktion des Insassenbestands mit einer extensiven Auslegung von Art. 86 Abs. 4 StGB begegnet werden könnte. Während die erste Frage bei strikter Beachtung der Verhältnismässigkeit zu bejahen ist, wird die systematische Anwendung von Art. 86 Abs. 4 StGB als Lösungsoption verworfen.

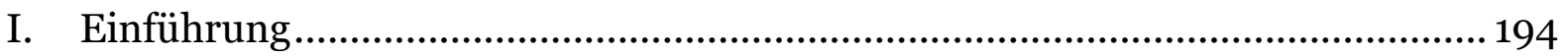

II. Temporäre Einschränkung der Therapiefrequenz.......................................... 195

1. Bedeutung von Art. 10a COVID-19-Verordnung 2 für

Psychotherapien in der Freiheit............................................................... 196

2. Unanwendbarkeit von Art. 10a COVID-19-Verordnung 2

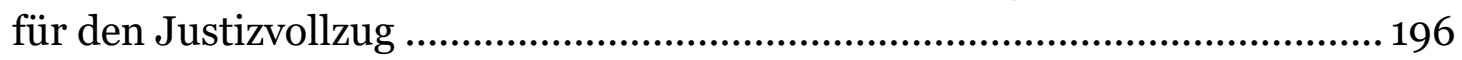

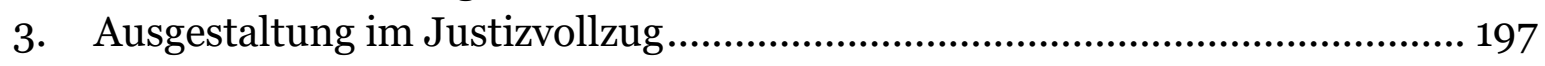

III. Extension der bedingten Entlassung nach Art. 86 Abs. 4 StGB ....................... 202

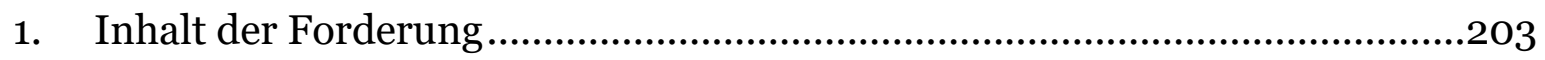

2. Kritische Würdigung ...................................................................203

IV. Abschliessende Bemerkungen ............................................................. 208

Zitiervorschlag: Thierry Urwyler/Thomas Noll/Astrid Rossegger, Corona-Prävention im Straf- und Massnahmenvollzug, in: sui-generis 2020, S. 193

URL: $\quad$ sui-generis.ch/130

DOI: $\quad$ https://doi.org/10.21257/sg.130

* Dr. iur Thierry Urwyler, Akademischer Mitarbeiter, Forschung und Entwicklung, Justizvollzug und Wiedereingliederung (Zürich) und Lehrbeauftragter Universität Luzern; Dr. med. und Dr. iur. Thomas Noll, Akademischer Mitarbeiter, Forschung und Entwicklung, Justizvollug und Wiedereingliederung (Zürich); PD Dr. rer. nat. Astrid Rossegger, Stv. Haupabteilungsleiterin, Forschung und Entwicklung/Co-Leiterin Arbeitsgruppe Forensische Psychologie Universität Konstanz. 


\section{Einführung}

1 Das Corona-Virus stellt die Schweiz vor menschliche, organisatorische und verfassungsrechtliche Herausforderungen. Weitreichende Eingriffe in die alltägliche Lebensgestaltung wurden vorgenommen, um die Ausbreitung des Virus zu verlangsamen und damit den notwendigen Schutz von Leib und Leben zu gewährleisten. ${ }^{1}$ Auch das Strafrecht im weiteren Sinne wird sich noch lange mit den Teilfragen rund um das Corona-Virus beschäftigen: Der Aufschub von Gerichtsverhandlungen/Einvernahmen, deren modifizierte Durchführung ${ }^{2}$, die Auslegung von Haftgründen 3 oder die Verhandlungsfähigkeit bei Risikogruppen 4 illustrieren den Einfluss der Pandemie auf das Verfahrensrecht. Weiter haben die Strafnormen der COVID-19Verordnung 2 materiell-strafrechtliche Spannungsfelder (Legalitätsprinzip) eröffnet.5 Sodann ist auch das Straf- und Massnahmenvollzugsrecht mit zahlreichen Herausforderungen konfrontiert. ${ }^{6}$ Die Kernfragen in dieser Rechtsmaterie lauten: Mit welchen präventiven Massnahmen wird einer Verbreitung des Virus

1 Vgl. Verordnung 2 über Massnahmen zur Bekämpfung des Coronavirus (COVID-19), (COVID19-Verordnung 2) vom 13. März 2020 (Stand am 4. April 2020).

2 Art. 1 Verordnung über Massnahmen in der Justiz und im Verfahrensrecht im Zusammenhang mit dem Coronavirus (COVID-19-Verordnung Justiz und Verfahrensrecht) vom 16. April 2020.

3 Urteil des Bundesgerichts 1B_112/2020 vom 20. März 2020 E. 3.3.

4 Urteil des Bundesstrafgerichts SN.2020.10 (SK.2019.45) vom 17. März 2020 E. 8.

5 «Strafrechtsprofessor Marcel Niggli tadelt Bundesrat - Corona-Bussen sind verfassungswidrig!», Blick am 6. April 2020.

6 Zur internationalen Berichterstattung auch der Überblick bei Prisonstudies; in der Schweiz stellvertretend: "Coronavirus hinter Gittern - Wenn Gefängnisse zu tickenden Zeitbomben werden», SRF Tagesschau vom 29. März 2020. im Justizvollzug entgegengewirkt und woraus ergibt sich die verfassungs- und völkerrechtliche Legitimität dieses Vorgehens?

2 Auf generell-abstrakter Ebene ist die Antwort klar: Der Staat trägt für die Gesundheit der inhaftierten Personen eine umfassende "menschen- und grundrechtliche Verantwortung» (Fürsorgepflicht, Schutzpflicht)7 und ist epidemiengesetzlich $^{8}$ zur Reduktion von Infektionsrisiken verpflichtet. Es sind daher alle gesetzlich vorgesehenen und verhältnismässigen Mittel zu verwenden, um die Ansteckung von inhaftierten Personen mit übertragbaren Krankheiten zu vermeiden. 9 Die Strategie von Justizvollzug und Wiedereingliederung des Kantons Zürich orientiert sich zu diesem Zweck an den nationalen (Bundesamt für Gesundheit [BAG], Konferenz der Kantonalen Justiz und Polizeidirektorinnen und -direktoren [KKJPD]) und internationalen Vorgaben (World Health Organization [WHO], Committee fort he Prevention of Torture [CPT]), wobei u.a. das Einhalten sozialer Distanz (sog «social distancing»), eingeschränkte Besuchsrechte (Art. 84 Abs. 2 StGB), der Einsatz von Trennscheiben, Sistierungen von Ausgang/ Urlaub und ultima ratio Isolationen

$7 \overline{\text { Übersicht über die einschlägigen Quellen bei Jörg }}$ Künzli/Alberto Achermann, Bekämpfung von Infektionskrankheiten im Freiheitsentzug, Jusletter 7. Mai 2007, Rz. 5 ff. und Zitat Rz. 24.

8 Künzli/Achermann (Fn. 7), Rz. 28; vgl. sodann Art. 30 der Epidemienverordnung.

9 Vgl. Künzli/Achermann (Fn. 7), Rz. 26 und 31; zentral sodann: EGMR, Guide on the case-law of the European Convention on Human Rights, Prisoners' rights, Updated on 31 December 2019, Rz. 122 ff. 
(Art. 78 lit. b StGB; Art. 90 Abs. 1 lit. b StGB) ${ }^{10}$ als Präventionsinstrumente eingesetzt werden. ${ }^{11}$ Darüber hinaus gibt es zwei weitere mögliche Massnahmen zur Reduktion der Infektionsgefahr, welche eine erhöhte juristische Komplexität aufweisen und eine differenzierte Betrachtung erfordern.

1. Erstens stellt sich die Frage, ob bzw. in welchem Rahmen es erlaubt oder gar geboten ist, nicht dringende psychotherapeutische Interventionen in ihrer Frequenz einzuschränken, um die Verbreitungsgefahr des Virus zu minimieren. Anlass zu dieser Abklärung ergibt sich aus Art.10a der COVID-19-Verordnung 2 (Fassung vom 21. März 2020). Diese Norm formuliert ein Verbot für nicht dringende Untersuchungen, Behandlungen und Therapien. Es wird zu prüfen sein, ob dieser Standard auch im Justizvollzug anwendbar ist bzw. ob sich analoge Standards aufdrängen.

2. Zweitens interessiert ${ }^{12}$, ob eine Reduktion des Insassenbestands in Einrichtungen des Freiheitsentzugs mit einer erweiterten Interpretation der ausserordentlichen bedingten Entlassung nach der Hälfte der Strafe

10 Zur Rechtfertigung dieses Vorgehens bei Tröpfcheninfektionen auch: Künzli/Achermann (Fn. 7), Rz. 35.

11 KKJPD, Zusammenfassung der nationalen und internationalen Rechtsgrundlagen und Empfehlungen zum Umgang mit COVID 19 in Anstalten des Freiheitsentzugs (Stand: 6. April 2020) Orientierungshilfe an die für den Justizvollzug zuständigen Stellen (zit. KKJPD, Zusammenfassung); sodann BAG, Neues Coronavirus: Massnahmen, Verordnung und Erläuterungen (zit. BAG, Neues Coronavirus)

12 Medienmitteilung von humanrights.ch «Coronavirus: Das Leben von Gefangenen steht über Sicherheitsinteressen» vom 23. März 2020. nach Art. 86 Abs. 4 StGB in zulässiger Weise umgesetzt werden könnte.

3 Mit der vorliegenden Publikation wollen die Autoren Antworten auf diese zwei Forschungsfragen formulieren und die darauf fussenden Entscheidungen von Justizvollzug und Wiedereingliederung im Kanton Zürich für Dritte transparent und kritisierbar machen. ${ }^{13}$

\section{Temporäre Einschränkung der Therapiefrequenz}

4 Wie einleitend erwähnt wurde, formuliert Art. 10a COVID-19-Verordnung ein Verbot von nicht dringenden Untersuchungen, Behandlungen und Therapien zwecks Eindämmung der Infektionsrisiken und Sicherstellung von medizinischen Ressourcen. Dieses Verbot tangiert auch Psychotherapien im Rahmen der allgemeinen Gesundheitsversorgung in der Freiheit (vgl. II.1). Allerdings wird sich zeigen, dass Art. 10a COVID-19Verordnung 2 den Justizvollzug nicht reguliert (vgl. II.2). Dennoch könnten analoge Schutzstandards zur Infektionsvermeidung im Justizvollzug angebracht sein. Solche Einschränkungen stehen jedoch in einem Spannungsfeld mit dem ebenso verfassungs- und konventionsrechtlichen Anspruch auf adäquate therapeutische Versorgung. Dieses Spannungsfeld ist nach Massgabe der Verhältnismässigkeit aufzulösen (vgl. II.3).

13 Die in diesem Beitrag erläuterten Rechtsfragen wurden während der Pandemie von der Geschäftsleitung von Justizvollzug und Wiedereingliederung an die Autoren in der Hauptabteilung «Forschung \& Entwicklung» herangetragen. Die vorliegenden Abhandlungen repräsentieren die Essenz der dafür notwendigen Rechtsabklärungen. 
1. Bedeutung von Art. 10a COVID-19Verordnung 2 für Psychotherapien in der Freiheit

5 Die COVID-19-Verordnung 2 (Fassung vom 21. März 2020) limitiert im Rahmen der allgemeinen Gesundheitsversorgung medizinische Untersuchungen, Behandlungen oder Therapien, welche nicht dringender Natur sind (Art. 10a Abs. 2-4). Nach Art. 10a Abs. 3 gelten jene Eingriffe als nicht dringend, die «zu einem späteren Zeitpunkt durchgeführt werden können, ohne dass bei der betroffenen Person Nachteile zu erwarten sind, die über geringe physische und psychische Beschwerden und Beeinträchtigungen hinausgehen» (lit. a) bzw. die «überwiegend oder vollständig ästhetischen Zwecken, der Steigerung der Leistungsfähigkeit oder dem Wohlbefinden dienen» (lit. b). In der Erläuterung zur COVID19-Verordnung 2 wurde dieser Schritt wie folgt begründet: Es soll zum einen vermieden werden, dass sich unnötig Menschenansammlungen bilden, und zum anderen sollen Kapazitäten der Gesundheitsversorgung nicht ohne zwingenden Grund gebunden werden. ${ }^{14}$

6 Die Berufsverbände haben diese Leitlinien aufgenommen, gleichzeitig aber ein extensives Dringlichkeitsverständnis stipuliert. Die Dachorganisation der psychiatrisch-psychotherapeutisch tätigen Ärzte und Ärztinnen der Schweiz (Foederatio Medicorum Psychiatricorum et Psychotherapeuticorum [FMPP]) stellt fest, dass psychische Dekompensationen nach Möglichkeit $\mathrm{zu}$ verhindern sind, um Hospitalisationen und die damit drohen-

Erläuterungen zur Verordnung 2 vom 13. März 2020 über Massnahmen zur Bekämpfung des Coronavirus (COVID-19-Verordnung 2), Fassung vom 25. März 2020, Stand 28. März 2020, S. 17. de Überlastung der Kliniken zu vermeiden. Folglich sollen psychotherapeutische Interventionen nach Möglichkeit weitergeführt werden, wobei die modernen Kommunikationsmittel zu nutzen sind, um die Ziele der bundesrätlichen Verordnung tunlichst gut umzusetzen. ${ }^{15}$ Ähnlich lauten die Vorgaben der Dachorganisation der in der Schweiz tätigen Psychologinnen und Psychologen (FSP): Therapien sollen nach Beurteilung der Dringlichkeit fortgesetzt werden, wobei Telefon-, Video- oder E-MailBehandlungen zu prüfen sind. ${ }^{16}$

\section{Unanwendbarkeit von Art. 10a COVID-19-Verordnung 2 für den Justizvollzug}

7 Grundsätzlich hätte der Bund im Rahmen des Epidemiengesetzes die Möglichkeit, Massnahmen für den Justizvollzug zu definieren. ${ }^{17}$ Mit Art. 10a der COVID19-Verordnung 2 (Pflichten der Gesundheitseinrichtungen) wurde jedoch nur die allgemeine Gesundheitsversorgung reguliert und nicht der Straf- und Massnahmenvollzug. ${ }^{18}$ Dieses Auslegungsresultat begründet sich wie folgt: Das Verbot von nicht dringenden medizinischen Untersuchungen, Behandlungen und Therapien gilt gemäss Art. 10a Abs. 2 COVID19-Verordnung 2 nur für die «Gesundheitseinrichtungen nach Artikel 6 Absatz 3 Buchstabe m». Unter diesen Begriff fallen insbesondere «Spitäler, Kliniken und Arztpraxen sowie Praxen und Einrichtungen von Gesundheitsfachpersonen nach Bundesrecht und kantonalem

15 Fachinformation der FMPP zur aktuellen Coronavirus-Pandemie (Stand 6. April 2020).

16 FAQ Coronavirus der FSP (nur für Mitglieder zugänglich; Stand 06. April 2020).

17 Künzli/Achermann (Fn. 7), Rz. 75 f.

18 So auch die Interpretation bei KKJPD, Zusammenfassung (Fn. 11). 
Recht» (Art. 6 Abs. 3 lit. m). Der Justizvollzug ist kein Teil dieser allgemeinen Gesundheitsversorgung. Vielmehr stellt er einen Teil der öffentlichen Verwaltung im Sinne von Art. 6 Abs. 3 lit. j COVID19-Verordnung 2 dar. Diese Interpretation wird durch die Ausführungen des BAG gestützt, welches unter der öffentlichen Verwaltung nach Art. 6 Abs. 3 lit. j Anstalten des Freiheitsentzugs explizit inkludierte und zusätzlich ausführte, es werde empfohlen, «in Anstalten des Freiheitsentzugs (Gefängnisse, Justizvollzugsanstalten) die Massnahmen zur Verminderung des Übertragungsrisikos und zur Bekämpfung des Coronavirus (COVID-19) an den Empfehlungen internationaler Organisationen, namentlich an den Empfehlungen der Weltgesundheitsorganisation (WHO) und des Europarats auszurichten.»19 Aus diesen Gründen ist Art. 10a COVID-19Verordnung 2 nicht auf den Justizvollzug anwendbar. Entsprechend obliegt es daher den Kantonen (Art. 1a COVID-19Verordnung 2), zu entscheiden, ob und in welchem Rahmen die Vorgaben von Art. 10a COVID-19-Verordnung 2 für der Justizvollzug Relevanz entfalten.

\section{Ausgestaltung im Justizvollzug}

8 Nachfolgend ist der Frage nachzugehen, ob sich im Justizvollzug trotz fehlender direkter Anwendbarkeit von Art. 10a COVID-19-Verordnung 2 gleichgelagerte Lösungsmodelle aufdrängen. Es wird sich zeigen, dass der Anspruch der inhaftierten Person auf Schutz vor Infektionskrankheiten für eine analoge Ausgestaltung spricht (vgl. II.3.a.aa). Gleichzeitig gerät dieser Lösungsansatz in einen Zielkonflikt mit dem Anspruch von Inhaftier-

BAG, Neues Coronavirus (Fn. 11). ten auf eine adäquate therapeutische Versorgung (vgl. II.3.a.bb). Dieses verfassungs- und konventionsrechtliche Spannungsverhältnis ist nach Massgabe der Verhältnismässigkeit aufzulösen (II.3.b).

\section{a) Konventions- und verfassungsrechtliches Spannungsverhältnis}

\section{aa)Anspruch der inhaftierten Person auf Schutz vor Infektionskrankheiten}

9 Der Staat tragt aufgrund Art. 2 und 3 EMRK (Art. 10 BV) eine umfassende verfassungs- und konventionsrechtliche Schutzpflicht für das Leben und die Gesundheit von inhaftierten Personen. ${ }^{20}$ Ansteckungen von inhaftierten Personen mit übertragbaren Krankheiten sind daher bestmöglich $\mathrm{zu}$ vermeiden. ${ }^{21} \mathrm{Nun}$ ist es so, dass durch Therapiesitzungen Infektionsrisiken ansteigen: Durch Wartebereiche, die räumlichen Verhältnisse (z.B. Raumgrösse oder Möglichkeit der Lüftung) usw. werden Personenzirkulation und -ansammlungen gefördert, was wiederum während Pandemien Infektionsrisiken steigen lässt. Angesichts dieser Ausgangslage wäre es mit Blick auf

20 Übersicht über die einschlägigen Quellen bei Künzli/Achermann, (Fn. 7), Rz. 5 ff. und Zitat Rz. 24; vgl. auch Rule 24 ff. der Nelson Mandela Rules, The United Nations Standard Minimum Rules for the Treatment of Prisoners.

21 Vgl. Künzli/Achermann (Fn. 7), Rz. 26 und 31; zur Rechtsprechung des EGMR sodann: EGMR, Guide on the case-law of the European Convention on Human Rights, Prisoners' rights, Updated on 31 December 2019, Rz. 122 ff.; Rule 30 der Nelson Mandela Rules, The United Nations Standard Minimum Rules for the Treatment of Prisoners; ebenso Freiheitsentzug, Die Empfehlungen des Europarates, Europäische Strafvollzugsgrundsätze, Godesberg 2007, Rz. 42.3. 
die staatliche Schutzpflicht plausibel, die Vorgaben der COVID-19-Verordnung 2 mutatis mutandis auf den Straf- und Massnahmenvollzug anzuwenden, da sich in diesem Rahmen (mindestens) dieselben Herausforderungen wie im Rahmen der allgemeinen Gesundheitsversorgung stellen.

\section{bb)Anspruch der inhaftierten Person auf therapeutische Ver- sorgung}

10 Eine Einschränkung des therapeutischen Angebots tangiert jedoch automatisch andere verfassungs- und konventionsrechtliche Verpflichtungen. Die Gewährleistung einer adäquaten medizinischen und psychotherapeutischen Versorgung gehört zu den positiven Leistungspflichten des Staates im Freiheitsentzug aufgrund des Folterverbots (Art. 3 EMRK, Art. 10 BV) und stellt bei inhaftierten Personen überdies eine Voraussetzung für die Gesetzmässigkeit des Freiheitsentzugs nach Art. 5 EMRK (Art. $31 \mathrm{BV}$ ) dar. ${ }^{22}$ Vor diesem Hintergrund dürften Vollzugsinstitutionen therapeutische Angebote nicht generell-abstrakt einschränken: Ein «therapeutic abandonment» wäre mit Art. 3 (Art. $10 \mathrm{BV}$ ) und 5 EMRK (Art. $31 \mathrm{BV}$ ) unvereinbar. ${ }^{23}$ Ebenso wäre eine generell-abstrakte Einschränkung des Therapieangebots auf einzelne Behandlungsformen (z.B. pharmakologische Behandlung) unzulässig. ${ }^{24}$ Aus-

$22 \overline{\text { Urteil des EGMR 34602/16 vom 21. Januar 202O }}$ (Strazimiri v. Albania) § 103 ff.; Urteil des EGMR 18052/11 vom 31. Januar 2019 (Rooman v. Belgium [GC]) § $146 \mathrm{ff}$. und $169 \mathrm{ff}$.

23 Urteil des EGMR 34602/16 vom 21. Januar 2020 (Strazimiri v. Albania) § $103 \mathrm{ff}$.

24 Urteil des EGMR 34602/16 vom 21. Januar 2020 (Strazimiri v. Albania) § 123 (So rügte der EGMR einen Staat zu Recht, der sich bei einer psychisch gestörten Person nur auf pharmakologische Interventionen beschränkt und ansonsten entge- serhalb dieser Extremkonstellationen bestehen jedoch gewisse Ermessensspielräume. ${ }^{25}$

\section{b) Auflösung des verfassungs- und konventionsrechtlichen Spannungsverhältnisses}

11 Aus dem Gesagten ergibt sich, dass die Ansprüche der inhaftierten Person auf Schutz vor Infektionen und auf die Gewährleistung einer angemessenen psychiatrischen und psychotherapeutischen Versorgung in einem sensiblen Spannungsverhältnis (Zielkonflikt) stehen. Nachfolgend wird ein Lösungsmodell präsentiert, das diesen Konflikt nach Massgabe der Verhältnismässigkeit auflöst.

\section{aa) Gewährleistung der medizi- nisch-psychologischen Grund- versorgung}

12 Für die Belange der allgemeinen Gesundheitsversorgung bildet das Äquivalenzprinzip den Ausgangspunkt aller Überlegungen. Danach muss die Gesundheitsfürsorge für inhaftierte Personen mit jener für Personen in Freiheit gleichwertig sein (Art. 75 StGB). ${ }^{26}$ Unter II.1 wurde dargelegt, dass die Berufsverbände die von Art. 10a COVID-19Verordnung 2 geforderte Dringlichkeit extensiv auslegen und Psychotherapien bestmöglich weiterführen. Folglich muss aufgrund des Äquivalenzprinzips auch die somatische und psychiatrische

gen seiner Pflicht kein therapeutisches Umfeld (inkl. Psychotherapie etc.) zur Verfügung gestellt hatte).

25 Urteile des EGMR 15669/13 and 76140/13 vom 17. März 2020 (Kosenko v. Russia) § $37 \mathrm{ff}$.

26 Benjamin Brägger, Gefängnismedizin, in: Benjamin Brägger (Hrsg.). Das schweizerische Vollzugslexikon Von der vorläufigen Festnahme zur bedingten Entlassung, Basel 2014. 
Grundversorgung für inhaftierte Personen bestmöglich gewährleistet werden. ${ }^{27}$ Dies gilt umso mehr, weil angesichts der Corona-bedingten Vollzugsrestriktionen Stressfaktoren vorliegen, welche psychische Leiden initiieren oder verstärken können (z.B. Depressivität, [Auto]Aggressivität etc.). ${ }^{28}$ In diesen Konstellationen überwiegen die Interessen der inhaftierten Person auf somatische und psychische Unversehrtheit das Interesse auf Vermeidung von Infektionsrisiken. Dabei ist anzumerken, dass die jeweiligen Interventionen zwecks Minimierung des Infektionsrisikos in Übereinstimmung der Hygienevorgaben der COVID19-Verordnung 2 (Art. 6 Abs. 4) bzw. den Empfehlungen des Bundesamts für Gesundheit vorgenommen werden.

\section{bb)Temporäre Frequenzein- schränkungen bei deliktpräven- tiven Therapien}

13 In einem zweiten Schritt stellt sich die Frage, ob Einschränkungen bei deliktpräventiven psychotherapeutischen Interventionen möglich wären. Solche finden sowohl im Rahmen von strafrechtlichen Massnahmen (Art. 56 ff. StGB) als auch freiwilligen Psychotherapien während des Strafvollzugs oder der Verwahrung statt und streben eine Reduktion des Rückfallrisikos an. Angesichts der verfassungs- und konventionsrechtlichen Ge-

27 KKJPD, Zusammenfassung (Fn. 11); Michael Liebrenz/Jonas Weber/Ueli Kieser/Roman Schleifer, COVID-19 Pandemie: Psychiatrische Versorgung von Inhaftierten, in: Jusletter 20. April 2020, Rz. 25.

28 Allgemein $\mathrm{zu}$ den Auswirkungen der Pandemie auf die psychische Gesundheit: Julio Torales et al., The Outbreak of COVID-19 Coronavirus and Its Impact on Global Mental Health, International Journal of Social Psychiatry 2020; vgl. sodann Liebrenz/Weber/Kieser/Schleifer (Fn. 27), Rz. 22. währleistungspflicht nach Art. 3 und 5 EMRK (bzw. Art. 10 und 31 BV) muss auch für sie die Maxime der bestmöglichen Aufrechterhaltung des therapeutischen Angebots gelten (vgl. II.3.a.bb).29 Die längerfristige Ausrichtung solcher Massnahmen öffnet sich jedoch der Betrachtung, dass nicht alle diese Interventionen «dringlich» im engeren Sinne sind (analoger Massstab zu Art. 10a COVIDVerordnung 2).30 Angesichts dieser Ausgangslage könnten einzelne Komponenten des deliktpräventiven Therapieangebots temporär eingeschränkt werden.

14 Im Rahmen des Zuständigkeitsbereichs des Psychiatrisch-Psychologischen Dienstes (PPD) in Justizvollzug und Wiedereingliederung wurde diese Massnahme ergriffen: Sowohl im stationären als auch im ambulanten Therapiesetting wurden Gruppensitzungen vorläufig sistiert. Milieutherapeutische Angebote werden für die Klienten in stationären Massnahmen beibehalten (unter Beachtung der Vorgaben des Bundes). Einzelsitzungen werden nach Indikation in reduzierter Frequenz weitergeführt bzw. dort wo möglich (am-

29 KKJPD, Zusammenfassung (Fn. 11).

3o Einen anderen als den hier argumentierten Weg gehen Liebrenz/Weber/Kieser/Schleifer (Fn. 27), Rz. 29, in dem sie die Dringlichkeit aufgrund des von ihnen angenommenen mangelnden Wirksamkeitsnachweis für rehabilitative Therapien annehmen. Dieses Argument ist jedoch angesichts des internationalen Forschungsstands zur Evaluation von deliktpräventiven Interventionen nicht haltbar; Vgl. Thierry Urwyler, Wirksamkeit therapeutischer Interventionen bei erwachsenen Sexualstraftätern: Implikationen der Evaluationsforschung auf die Verhältnismässigkeit therapeutischer Massnahmen, in: 20 Jahre Amt für Justizvollzug Zürich - eine Festschrift, Schweizerische Zeitschrift für Kriminologie (SZK) 2019 (Sonderband), 100 ff.; generell sodann Jérôme Endrass et al., (Hrsg.), Interventionen bei Gewaltund Sexualstraftätern, Risk-Management, Methoden und Konzepte der forensischen Therapie, Berlin 2013. 
bulante Behandlungen in Freiheit), via Telefon-/Internetdienste durchgeführt. Bei inhaftierten Personen, die kurz vor der (bedingten) Entlassung stehen, werden Einzelsitzungen möglichst mit normaler Intensität weitergeführt, um ein geordnetes Übergangsmanagement sicherzustellen.

\section{cc) Verfassungs- und konventions- rechtliche Zulässigkeit der Ein- schränkung}

15 Die vorangehenden Einschränkungen greifen in das Recht der inhaftierten Person auf therapeutische Versorgung ein (II.3.a.aa) und müssen daher rechtsstaatlichen Eingriffsanforderungen (Art. $36 \mathrm{BV}$ ) standhalten. Hinsichtlich der gesetzlichen Grundlage könnten grundsätzlich allgemeine Vorschriften zum Strafvollzug (Art. 75 Abs. 1 StGB) oder Art. $30 \mathrm{EpV}$ angerufen werden: Beide dieser Normen enthalten jedoch keine explizite Formulierung, welche eine temporäre Therapieeinschränkung legitimieren würde und wären daher von der Normbestimmtheit (und -stufe bei Art. $30 \mathrm{EpV}$ ) als problematisch einzustufen, zumal die Einschränkungen deliktpräventiver Therapien durchaus eine gewisse Intensität aufweist. Auf kantonaler Ebene könnte $\S 23$ a StJVG als Gesetzesgrundlage angerufen werden. Diese Norm erwähnt zwar die hier diskutierten Therapieeinschränkungen nicht explizit, berechtigt aber in nicht abschliessender Aufzählung zu Sicherheits- und Schutzmassnahmen. Selbst wenn man strengste Anforderungen an die Normdichte als gerechtfertigt halten würde, wäre die ausserordentliche Lage nach Epidemiengesetz geradezu ein idealtypischer Anwendungsfall der polizeilichen Generalklausel, welche zur
Rechtfertigung der hier zu betrachtenden Eingriffe angerufen werden kann. ${ }^{31}$

16 Auf Basis dieser Feststellung ist zu prüfen, ob den Einschränkungen hinreichende öffentliche Interessen zugrunde liegen. Dies ist der Fall. Vorliegend geht es um den Schutz von hochwertigen Rechtsgütern von inhaftierten Personen vor unmittelbaren Gefahren. Zum einen sind die Inhaftierten vor Infektionen zu schützen (vgl. II.3.a.aa) und zum anderen muss sichergestellt sein, dass auch bei einer Ausbreitung des Virus eine adäquate psychotherapeutische Versorgung durch das Therapiepersonal sichergestellt wäre (vgl. II.3.a.bb).

17 Darüber hinaus halten die temporären Frequenzeinschränkungen von deliktpräventiven Psychotherapien einer Verhältnismässigkeitsprüfung stand. Durch die Einschränkungen der Therapiefrequenz werden sowohl die Personenzirkulation als auch -konzentration in bestimmten Bereichen von Justizvollzugseinrichtungen minimiert und das Risiko einer Infektion mit dem Corona-Virus für inhaftierte Personen gesenkt (Eignung bzgl. Schutz von Leib und Leben). Zeitgleich kann sichergestellt werden, dass die zuständigen Fachpersonen (PsychotherapeutInnen.) bei einer grösseren Verbreitung der Infektionskrankheit (auch unter den Mitarbeitenden) eine adäquate psychotherapeutische Versorgung auf lange Frist aufrechterhalten können (Eignung bzgl. Gewährleistung Therapieressourcen).

${ }_{31} \overline{\text { Vgl. zum Rechtsinstitut: Andreas Zünd/Christoph }}$ Errass, Die polizeiliche Generalklausel in der Schweiz. 
Sodann besteht die Pflicht, diese Einschränkungen auf das Minimum zu beschränken (Erforderlichkeit; Übermassverbot). Die unter II.3.b.bb erwähnten Limitationen beachten diese Leitplanken: Wo keine Konsultationen in Anwesenheit bei genügend grossen und gelüfteten Räumen (allenfalls hinter einer Trennscheibe) möglich sind, sollen telefonische/digitale Kommunikationswege genutzt und erst dann zu Sistierungen von Therapiesitzungen geschritten werden. ${ }^{2}$ Auch diese Anforderungen werden im Status Quo eingehalten. Wo möglich, werden digitale Kontaktmöglichkeiten eingesetzt. Einzelsitzungen im Freiheitsentzug werden in ihrer Frequenz eingeschränkt, aber nicht aufgehoben, womit der Eingriff auf das sachlich Erforderliche eingeschränkt wird. Einzig für Gruppensitzungen ist aufgrund der akzentuierten Infektionsproblematik eine temporäre Sistierung das sachlich mildeste Mittel. Zur Gewährleistung der Erforderlichkeit in zeitlicher Hinsicht werden die einschränkenden Massnahmen zudem befristet (vorläufig bis am 15. Mai 2020 bei Justizvollzug und Wiedereingliederung des Kantons Zürich) und laufend an neue Umstände und/oder Vorgaben nationaler und internationaler Organisationen (WHO, BAG-Richtlinien und -Empfehlungen etc.) angepasst. Damit beschränken sich die Eingriffe auf das sachlich, zeitlich, räumlich und personell Notwendige.

19 Die Massnahmen sind auch verhältnismässig im engeren Sinne. Auf Seiten der inhaftierten Person ist zu berücksichtigen, dass aus den Einschränkungen der

$32 \overline{\text { KKJPD, Zusammenfassung (Fn. 11); zu digitalen }}$ Therapiemöglichkeiten auch Liebrenz/Weber/ Kieser/Schleifer (Fn. 27), Rz. 30 ff.
Therapiedichte potenzielle Negativeffekte für die betroffenen Personen resultieren. Konkret besteht die Möglichkeit, dass betroffene Personen erst später Therapiefortschritte erzielen, was sich negativ auf ihren Vollzugsverlauf (bedingte Entlassung usw.) auswirken kann.33 Auf der anderen Seite ist das wohlverstandene Interesse derselben (und aller anderen) inhaftierten Personen zu beachten, nicht mit dem CoronaVirus angesteckt zu werden, womit Gefahren für Leib und Leben einhergehen. Darüber hinaus besteht ein Interesse aller inhaftierten Personen darin, auch bei einer Eskalation der Infektionsrate (inkl. Erkrankungsfällen beim Therapiepersonal) langfristig therapeutische Angebote nutzen zu können. In einer Gesamtwürdigung überwiegen diese öffentlichen Interessen das private Interesse der inhaftierten Person auf umfassende und konstante Gewährleistung des deliktpräventiven Therapieangebots während der Pandemiephase. Die Verbreitung des Virus in Einrichtungen des Justizvollzugs hätte potenziell gravierende (z.T. irreversible34) Gesundheitsfolgen für zahlreiche Inhaftierte und für Personen in ambulanter Behandlung in Freiheit und könnte für Einzelne sogar zum Tod führen. Würde im Verbreitungsfall zudem das therapeutische Angebot an deliktpräventiven Therapien bei vermehrten Krankheitsfällen unter dem Therapiepersonal zusammenbrechen, wären ultimativ grössere Einschnitte in das Recht auf therapeutische Angebote zu befürchten. 35

33 «Die Gefangenen können jetzt skypen», Tagesanzeiger vom 27. März 2020.

34 «Video reveals lung damage in US coronavirus patient: People need to take this seriously», CNN health vom 27. März 2020.

35 Im vorliegenden Fall entsteht folglich die besondere Situation, dass durch eine temporäre Ein- 
Angesichts dieser Ausgangslage sind die temporären Einschränkungen eine verhältnismässige Folge der staatlichen Schutzpflicht während einer Notlage und halten einer menschenrechtlichen Betrachtung stand.

20

Abschliessend drängen sich folgende Bemerkungen zum zuvor erwähnten Risiko von therapeutischen Negativeffekte auf. Sofern die Einschränkungen kurzfristiger Natur sind (z.B. ein Unterbruch von wenigen Wochen), sollte sich das Risiko für diese Effekte in überschaubarem Ausmass halten. Erst wenn die Notlage und die korrespondierenden Einschränkungen länger anhalten, wäre ein gehäuftes Vorkommen dieser Negativfolgen wahrscheinlich. Wenn es im Einzelfall zu therapeutischen Verzögerungen oder gar Rückschritten kommt, müssten diese für die inhaftierte Person entstehenden Nachteile bei der Verhältnismässigkeitsprüfung zugunsten der inhaftierten Person (z.B. Fortführung der Massnahme, bedingte Entlassung etc.) berücksichtigt werden.

\section{Extension der bedingten Entlas- sung nach Art. 86 Abs. 4 StGB}

Unter diesem Titel soll auf die zweite Forschungsfrage eingegangen und geprüft werden, ob unter Art. 86 Abs. 4 StGB eine extensivere Entlassungspraxis in Pandemiezeiten möglich wäre. Einleitend wurde bereits erwähnt, dass in Einrichtungen des Freiheitsentzugs erhöhte Infektionsrisiken bestehen. Daher ist eine Reduktion der Anzahl von inhaftierten

schränkung des psychotherapeutischen Angebots die langfristige Gewährleistung des Rechts auf therapeutische Versorgung nach Art. 5 EMRK und Art. 31 BV gewährleistet werden kann.
Personen ein wichtiges Mittel in einer Pandemie-Gesamtstrategie. 36 Komplizierter wird die Beantwortung der Frage, mit welchen konkreten Instrumenten diese Forderung umzusetzen ist. Sofern Ermessensspielräume wie bei der Bestimmung des Termins für den Antritt einer Freiheitsstrafe37 bzw. Ersatzfreiheitstrafe38 genutzt werden, scheint dies sinnvoll, sofern die Haftzwecke (insb. Rückfallrisiko) es erlauben. 39 Ebenso kann die bedingte Entlassung nach Art. 86 Abs. 1 StGB oder der Einsatz besonderer Vollzugsformen (E-Monitoring etc.) den erwünschten Effekt herbeiführen, dass sich weniger Personen in Haft befinden. Dabei handelt es sich jedoch nicht um eine Corona-spezifische Adaption der massgeblichen Normen, sondern schlicht um eine erwünschte Nebenfolge einer auch ausserhalb von Krisenzeiten anwendbaren korrekten Gesetzesauslegung. Von diesen juristischen Gestaltungsmöglichkeiten abgesehen sind aber auch Vorschläge formuliert worden, die einer genaueren Betrachtung bedürfen: Einer davon betrifft die Forderung nach einer extensiven Auslegung von Art. 86 Abs. 4 StGB. 40

36 «Wie reagiert der Justizvollzug auf COVID-19?», Schweizerisches Kompetenzzentrum für Justizvollzug; CPT, Statement of principles relating to the treatment of persons deprived of their liberty in the context of the coronavirus disease (COVID19) pandemic issued on 20 March 2020.

37 «Wie sich die Corona-Krise auf den Justizvollzug auswirkt», Rundfunk Berlin-Brandenburg (RBB), 17. März 2020.

38 Vgl. hierzu etwa «Berliner Justiz setzt Ersatzfreiheitsstrafe aus», RBB, 14. März 2020.

39 «Coronavirus hinter Gittern - Wenn Gefängnisse zu tickenden Zeitbomben werden» (Fn. 6); «Wie sich die Corona-Krise auf den Justizvollzug auswirkt» (Fn. 37); «Wegen Coronavirus: In diesem Kanton werden Kleinkriminelle nicht mehr verhaftet», watson, 5. April 2020.

40 Medienmitteilung humanrights.ch (Fn. 12). 


\section{Inhalt der Forderung}

22 In einer Medienmitteilung vom 23. März 2020 äusserte humanrights.ch (eine Menschenrechtsorganisation) ihre Besorgnis über den Schutz der Gesundheit von inhaftierten Personen während der Pandemie und formulierte mehrere Anliegen. Viele davon verdienen Zustimmung (Einhaltung der Verhältnismässigkeit, äquivalenter Schutz etc.). Ebenso wurde gefordert, dass die Anzahl Gefangener in Einrichtungen des Freiheitsentzugs reduziert werden solle. Als eines der möglichen Mittel wurde dabei die ausserordentliche bedingte Entlassung nach Art. 86 Abs. 4 StGB genannt. Diese Norm hat den folgenden Wortlaut: "Hat der Gefangene die Hälfte seiner Strafe, mindestens aber drei Monate verbüsst, so kann er ausnahmsweise bedingt entlassen werden, wenn ausserordentliche, in der Person des Gefangenen liegende Umstände dies rechtfertigen.». Gemäss humanrights.ch soll während der Corona-Pandemie «die Notwendigkeit des Schutzes der Gesundheit bei besonders gefährdeten Personen als 'ausserordentliche, in der Person des Gefangenen liegende Umstände' ausgelegt» und folglich inhaftierte Personen systematisch nach Verbüssung der Hälfte der Strafe entlassen werden. ${ }^{41}$

\section{Kritische Würdigung}

23 Der Ratio des erwähnten Ansinnens, den Insassenbestand in Vollzugseinrichtungen so tief wie möglich zu halten und damit das Leben der Inhaftierten zu schützen, ist vollumfänglich beizupflichten. Dennoch muss der darauf gestützten Forderung nach einer extensiven Interpretation von Art. 86 Abs. 4 StGB wider-

41 Medienmitteilung humanrights.ch (Fn. 12). sprochen werden. Es wird sich zeigen, dass Art. 86 Abs. 4 nicht unabhängig von Art. 86 Abs. 1 StGB ausgelegt werden kann (III.2.a) und es bei generellen Infektionsrisiken am Personenbezug im Sinne von Art. 86 Abs. 4 StGB fehlt (III.2.b). Hinzu kommen Herausforderungen der Umsetzung, welche der Vollständigkeit halber $\mathrm{zu}$ nennen sind (III.2.c). Abschliessend gilt es aufzuzeigen, welche juristischen Alternativen zielführender wären (III.2.d).

\section{a) Legalprognose als Basisvoraussetzung}

24 In einem ersten Schritt ist auf den systematischen Zusammenhang von Art. 86 Abs. 4 StGB und Art. 86 Abs. 1 StGB einzugehen. Der Gesetzgeber stellte klar, dass die ausserordentliche bedingte Entlassung nach Art. 86 Abs. 4 StGB gleich wie die ordentliche bedingte Entlassung im Rahmen einer Gesamtwürdigung eine günstige Legalprognose 42 i.S.v. Art. 86 Abs. 1 StGB voraussetzt.43 Diese gesetzgeberischen Leitlinien wurden von der Rechtsprechung zurecht bestätigt. 44 Die bedingte Entlassung und die mit ihr verfolgte Wiedereingliederung ist kein Selbstzweck, sondern «sondern auch ein Mittel, um die Allgemeinheit vor neuen

42 Präziser noch: Die Abwesenheit einer doppelt negativen Differenzialprognose $=$ die Abwägung zwischen der Prognose bei Vollverbüssung der Strafe versus die Prognose bei bedingter Entlassung unter Bewährungshilfe und Auflagen (BSKKoller, Art. 86 n 16).

43 Botschaft vom 21. September 1998 zur Änderung des Schweizerischen Strafgesetzbuches (Allgemeine Bestimmungen, Einführung und Anwendung des Gesetzes) und des Militärstrafgesetzes sowie zu einem Bundesgesetz über das Jugendstrafrecht, 2120 «Eine günstige Resozialisierungsprognose dieser Art wird auch vorausgesetzt für die ausserordentliche bedingte Entlassung nach der Hälfte der Strafdauer».

44 Urteil des Bundesgerichts 6B_240/2012 vom 4. Dezember 2012 E. 2.1. 
Straftaten zu schützen».45 Daraus ergibt sich, dass eine bedingte Entlassung nach der Hälfte der Strafe zumindest bei jenen Personen nicht in Frage käme, welche eine ungünstige Prognose i.S.v Art. 86 Abs. 1 StGB aufweisen. Die unter III.1 geforderte systematische Entlassung aller verurteilten Personen fällt daher bereits aus diesen Gründen für einen Teil der inhaftierten Personen ausser Betracht.

In diesem Zusammenhang drängen sich einige Bemerkungen zur sensiblen Abwägung im Rahmen von Art. 86 StGB auf. Die Forderungen nach einer extensiveren Entlassungspraxis wurden von der unter dem Titel «Das Leben von Gefangenen steht über Sicherheitsinteressen» vorgebracht. Im Text wurde dazu weiter ausgeführt, «das Recht auf Leben und Gesundheit der Gefangenen und der Mitarbeitenden» müsse höher gestellt werden «als abstrakte Sicherheitsinteressen».46 Die gewählten Formulierungen zeichnen ein verzerrtes Bild von den Entscheidungsprozessen im Straf- und Massnahmenvollzug im Rahmen der bedingten Entlassung. Nicht näher mit Vollzugsfragen befasste Lesende könnten bei den erwähnten Formulierungen zur Interpretation gelangen, dass im Status Quo der Justizvollzug die Gesundheit und das Leben von Gefangenen unspezifischen Sicherheitsabwägungen opfert.

Eine solche Wahrnehmung würde jedoch nicht den Tatsachen entsprechen. Es werden in Erfüllung der staatlichen Schutzpflicht zahlreiche Massnahmen ergriffen, damit eine Verbreitung des Virus nicht stattfindet und inhaftierte Per-

45 Urteil des Bundesgerichts 6B_32/2019 vom 28. Februar 2019 E. 2.10; BGE 124 IV 193 E. 3.

46 Medienmitteilung humanrights.ch (Fn. 12). sonen eine äquivalente Gesundheitsfürsorge erfahren (vgl. I, II sowie III.2.d). Ausschlaggebend ist im vorliegenden $\mathrm{Zu}$ sammenhang sodann die Feststellung, dass bei der für eine bedingte Entlassung anfallenden Gesamtwürdigung nicht abstrakte Sicherheitserwägungen, sondern konkrete Risiken für Leib, Leben und andere hochwertige Rechtsgüter von Dritten zu berücksichtigen sind, wobei das Rückfallrisiko freilich je nach inhaftierter Person variiert. Gemäss Angaben des Bundesamts für Statistik werden ca. 35\% der entlassenen Personen innert drei Jahren wieder mit einem Verbrechen oder Vergehen rückfällig. 47 Ebenso konnten Untersuchungen im Kanton Zürich aufzeigen, dass ca. $11-15 \%$ der entlassenen Straftäter im Beobachtungszeitraum (Beobachtungszeitraum im Schnitt 7.9 Jahre) mit einer schweren Gewaltoder Sexualstraftat rückfällig wurden. 48 Sodann entspricht es einem kriminologischen Erfahrungswert, dass die Zeit unmittelbar nach der Entlassung als eigentliche Hochrisikophase gilt, in der sich die meisten Rückfälle ereignen.49 Diese Aspekte wären neben anderen Faktoren im Rahmen einer der Gesamtwürdigung nach Art. 86 Abs. 1 StGB zu berücksichtigen. Aus diesen Bedingungen geht hervor, dass der Entscheid über eine bedingte Entlassung eine komplexe Abwägung zwischen Resozialisierungszielen und Si-

$47 \overline{\text { Zahlen des BFS über die Rückfallrate innerhalb }}$ von drei Jahren nach einer Entlassung für ein Verbrechen oder ein Vergehen, nach demografischen Merkmalen und Anzahl Vorstrafen vom 3. Dezember 2019.

48 Katharina Seewald et al., Effectiveness of a riskneed-responsivity-based treatment program for violent and sexual offenders: Results of a retrospective, quasi-experimental study, Legal and Criminological Psychology, 2018, 23, 85-99.

49 Thierry Urwyler et al., Die Führungsaufsicht und ihre potenziellen Alternativen, in: Jusletter 3. Februar 2020, Rz. 3. 
cherheitsinteressen der Allgemeinheit darstellt. Es wäre unzulässig, hochwertige Rechtsgüter von Dritten durch undifferenzierte Entlassungspraxen zu gefährden, wenn im Einzelfall ein ungünstiges Risikoprofil bei einer inhaftierten Person vorliegt. Es wäre wünschenswert gewesen, dass die hier reflektierte Forderung (III.1) den sensiblen Risiko-Abwägungsvorgang im Rahmen der bedingten Entlassung stärker zum Ausdruck gebracht hätte.

\section{b) Fehlender Personenbezug allgemeiner Infektionsrisiken}

Eine bedingte Entlassung zur Strafhälfte käme nach dem Gesagten allenfalls für jene Personen in Frage, welche eine günstige Legalprognose im Sinne von Art. 86 Abs. 1 StGB aufweisen. Auf Basis dieser Feststellung bleibt zu prüfen, ob die einschlägigen Tatbestandsmerkmale von Art. 86 Abs. 4 StGB während der Pandemie vorliegen. Legitimationsgrundlage für eine Entlassung nach der Strafhälfte sind nach dem Gesetzestext «ausserordentliche, in der Person des Gefangenen liegende Umstände.» Damit hat der Gesetzgeber zwar eine offene Formulierung gewählt.5o Unbestritten war jedoch immer, dass es sich um personenbezogene Umstände der inhaftierten Person selbst handeln muss; der Tod eines Familienangehörigen oder ein statistischer Rückgang der Delikte, welche die Verurteilung des Betroffenen veranlasst haben, sollten nach der Vorstellung des Gesetzgebers keine ausserordentlichen Gründe darstellen.51 Beispiele für

\footnotetext{
Botschaft (Fn. 43).

Botschaft (Fn. 43); vgl. auch Daniel Jositsch/ Gian Ege/Christian Schwarzenegger, Strafrecht II, Strafen und Massnahmen, 9. A., Zürich 2018, S. 252; Cornelia Koller, Art. 86 N 18, in: Niggli
}

personenbezogene Umstände waren demgegenüber irreversible Krankheiten der inhaftierten Person oder wenn sich eine inhaftierte Person in einer Katastrophenlage für einen besonders gefährlichen Einsatz zur Verfügung stellt genannt. ${ }^{2}$

28 Das Schrifttum - sofern es sich über die Inhalte der Materialien und Gerichtsurteile hinaus zur Frage äussert53 - kritisierte in Teilen die moralisierenden Fallgruppen des Gesetzgebers und stellte sich überwiegend auf den Standpunkt, dass spezialpräventive Gesichtspunkte den Ausschlag geben sollen.54 Auch bei dieser Interpretation bleibt indes der Personenbezug der Norm das kennzeichnende Merkmal, da letztlich die Individualprognose einer konkreten inhaftierten Person anwendungsbestimmend für

Alexander Marcel/Wiprächtiger Hans (Hrsg.), Basler Kommentar, Strafrecht, Strafgesetzbuch, Jugendstrafgesetz, 4. A., Basel 2018.

52 Botschaft (Fn. 43); vgl. auch Günther Stratenwerth, Schweizerisches Strafrecht. Allgemeiner Teil II: Strafen und Massnahmen, Bern 2006, § 4 N 69 (besonders verdienstliches Verhalten).

53 Keine über Materialien und Urteile hinausgehenden Ausführungen enthalten beispielsweise Stefan Heimgartner, Art. $86 \mathrm{~N} \mathrm{12}$, in: Andreas Donatsch (Hrsg.) StGB/JStG Kommentar, 20. A., Zürich 2018; Michel Dupuis et al., Petit Commen-

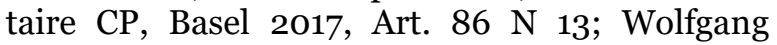
Wohlers, Art. $86 \mathrm{~N} \mathrm{4}$, in: Wolfgang Wohlers/ Gunhild Godenzi/Stephan Schlegel, Schweizerisches Strafgesetzbuch, Handkommentar, 4. A., Bern 2020.

54 Stefan Trechsel/Peter Aebersold, Art. 86 N 16, in: Stefan Trechsel/Marc Pieth (Hrsg.), Schweizerisches Strafgesetzbuch, Praxiskommentar, 3. A., Zürich/St.Gallen 2017; André Kuhn, Art. 86 N 8, in: Roth Robert/Moreillon Laurent (Hrsg.), Commentaire Romand, Code pénal I, Art. 1-110, Basel 2009; Die Praxis der bedingten Entlassung aus dem Strafvollzug, Eine empirische Studie zur Anwendung des Art. 86 StGB in den Kantonen Bern, Freiburg, Luzern und Waadt, Berlin/Bern 2019, 372. Cornelia Koller, Art. 86 N 18, in: Niggli Alexander Marcel/Wiprächtiger Hans (Hrsg.), Basler Kommentar, Strafrecht, Strafgesetzbuch, Jugendstrafgesetz, 4. A., Basel 2018. 
Art. 86 Abs. 4 StGB wäre (d.h. nur wenn die Prognose wegen a.o. Umständen günstig ausfiele, könnte die bedingte Entlassung zur Strafhälfte gewährt werden). Schliesslich knüpfen auch die Richtlinien der Strafvollzugskonkordate, die als Auslegungshilfen bei der Interpretation von Art. 86 Abs. 4 StGB beigezogen werden können55, an personenbezogene Umstände an: Genannt werden z.B. der Gesundheitszustand eines Insassen, die besondere Betroffenheit der inhaftierten Person durch die Folgen der Tat, durch aussergewöhnliche Entbehrungen erfolgte Verbesserungen der Legalprognose oder die Erfüllung finanzieller Pflichten aus der Verurteilung/dem Vollzugsverfahren. 56 Sodann hat auch die Rechtsprechung immer auf konkrete Personenumstände Bezug genommen und Art. 86 Abs. 4 StGB in die Nähe eines Gnadenakts (Begnadigung -> Einzelfallgerechtigkeit) gestellt. 57

55 Dabei sind die Richtlinien freilich nicht bindend (a.M. scheinbar das Verwaltungsgericht des Kantons Zürich VB.2010.00459 vom 11. November 2010 E. 3). Der Position des Verwaltungsgerichts muss jedoch widersprochen werden. Bei der Auslegung von Art. 86 Abs. 4 StGB sind die normalen Auslegungsgrundsätze zu beachten, d.h. es ist der Sinn der Norm durch grammatikalische, historische, teleologische und systematische Auslegung zu ermitteln. In diesem Rahmen können konkordatliche Richtlinien nur - aber immerhin - Hilfen bei der Auslegung von Normen mit unklarem Normgehalt sein.

56 Ostschweizer Strafvollzugskommission, Richtlinien betreffend die bedingte Entlassung aus dem Strafvollzug vom 7. April 2006, Rz. 2.2; Art. 3 Richtlinie der Konkordatskonferenz des Strafvollzugskonkordats der Nordwest- und Innerschweizer Kantone betreffend die bedingte Entlassung aus dem Strafvollzug vom 26. Oktober 2018 (enthalten nur den Gesundheitszustand und die schwere, nach der Verurteilung entstandene Betroffenheit).

57 Urteil des Bundesgericht 6B_240/2012 vom 4. Dezember 2012 (nicht erfüllt); Urteil des Bundesgerichts 6B_715/2014 vom 27. Januar 2015, (nicht erfüllt); Urteil des Bundesgerichts 6B_891/2008 vom 20. Juni 2009 (nicht erfüllt);
29 Aus dem Gesagten geht hervor, dass die von Art. 86 Abs. 4 StGB genannten ausserordentlichen Umstände eine konkrete inhaftierte Person betreffen müssen (Krankheit, besondere Verdienste, insb. Verbesserung Legalprognose). Bei der Corona-Pandemie handelt es sich jedoch nicht um solche individuelle Besonderheit, die eine ausserordentliche bedingte Entlassung rechtfertigen könnten, sondern um ein Phänomen mit Systemrelevanz, das alle in der Schweiz lebenden Menschen betrifft und für den Grossteil der Personen (auch der Inhaftierten) als Gesundheits- bzw. (Risikogruppen) Lebensrisiko in Erscheinung tritt.58 Zwar wird nicht bestritten, dass eine CoronaInfektion mit einem erhöhten Risiko für die Gesundheit (z.B. Lungenentzündung) und bei Risikogruppen für das Leben einhergeht und dass die Infektionsgefahr in Einrichtungen des Freiheitsentzugs tendenziell erhöht ist.59 Es bleibt aber bei einem für alle Inhaftierten bestehenden Risiko (probabilistische Grösse) und keinem auf eine konkrete inhaftierte Person bezogenen ausserordentlichen Umstand (z.B. eine irreversible Krankheit). Eine bedingte Entlassung zur Strafhälfte könnte daher erst dann diskutiert werden, wenn eine Person sich mit dem Vi-

Urteil des Verwaltungsgerichts Zürich VB.2010.00459 vom 11. November 2010, (nicht erfüllt); Urteil des Verwaltungsgerichts Zürich VB.2019.00165 vom 8. Juli 2019 (nicht erfüllt).

${ }_{58}$ Zur Risikoausprägung vgl. Liebrenz/Weber/ Kieser/Schleifer (Fn. 27), Rz. 1.

59 Liebrenz/Weber/Kieser/Schleifer (Fn. 27), Rz. 19; nach Kenntnisstand der Autoren ist jedoch nicht geklärt, wie stark die relative Risikoerhöhung (Ansteckungsrate Allgemeinbevölkerung v. Inhaftierte) gegenüber den Personen in Freiheit tatsächlich ausfällt. Dabei fällt eine exakte Risikoquantifizierung mangels gesicherter Datenlage schwer. Während kaum strittig sein dürfte, dass ein erhöhtes Ansteckungsrisiko besteht, ist unklar, wie hoch diese relative Risikoerhöhung gegenüber Personen in Freiheit ausfällt. 
rus infiziert und sich der Gesundheitszustand rapide verschlechtert. Dann läge der erforderliche Personenbezug des ausserordentlichen Umstands für den kranken Insassen vor. Allerdings wäre Art. 86 Abs. 4 StGB u.E. auch in diesen Konstellationen nicht einschlägig, da die Mehrzahl von Krankheitsverläufen bei COVID19 mit einer Gesundung enden und die Genesenen zu alten Kräften zurückkehren (mangelnde Irreversibilität i.S.v. Art. 86 Abs. 4 StGB). Zielführender wären daher bei dieser Sachlage Verlegungen in Spitäler und spezialisierte Einrichtungen (vgl. III.2.d).

Der einzige den Autoren vorstellbare Anwendungsfall für Art. 86 Abs. 4 StGB würde eintreten, wenn aufgrund der Corona-Krise ein Zusammenbruch des Gesundheitssystems wegen mangelnder Personalressourcen droht. Würden sich in einer solchen Sachverhaltskonstellation unter den inhaftierten Personen solche mit medizinischer/pflegerischer Ausbildung und akzeptablem extramuralen Risikoprofil befinden und wären diese bereit, Einsätze in den Spitälern etc. zu leisten, könnte sich Art. 86 Abs. 4 StGB als einschlägig erweisen. Sieht man von diesem Sonderfall ab, wäre die ausserordentliche bedingte Entlassung nach Art. 86 Abs. 4 StGB nicht das richtige Mittel, um während der Pandemie die angestrebte Reduktion des Insassenbestands in Einrichtungen des Freiheitsentzugs herbeizuführen. ${ }^{6}$

6o Wenn überhaupt, müsste man generell-abstrakte Lösungen für alle Gefangenen ausserhalb der Strukturen der Art. 75 ff. StGB unter dem Gesichtspunkt der Amnestie (Art. 384 StGB) betrachten, wobei es sich bei einer Infektionswelle wohl um einen atypischen Fall einer Amnestie handeln würde. Freilich wäre für eine solche die Bundesversammlung zuständig (Art. 384 Abs. 1 StGB).

\section{c) Herausforderungen der Umsetzung}

31 Abrundend sollen einige Umsetzungsherausforderungen der systematischen Anwendbarkeit von Art. 86 Abs. 4 StGB diskutiert werden. Dabei gilt es zu betonen, dass die nachfolgend genannten Aspekte keine Grundlagen darstellen, um bedingte Entlassungen aus dem Strafvollzug abzulehnen. Liegen die juristischen Voraussetzungen nach Art. 86 Abs. 4 StGB vor, ist die bedingte Entlassung zu gewähren. Dennoch ist es abgebracht, zur vollständigen Erfassung der Problematik die Konsequenzen einer systemischen Entlassungspraxis nach Art. 86 Abs. 4 StGB zu skizzieren: Würde die bedingte Entlassung nach der Strafhälfte während der Corona-Krise zur Regel, würde sich die Frage stellen, auf welche Weise ein geordnetes Übergangsmanagement in kurzer Frist bereitgestellt werden kann. Gerade bei Langzeitinhaftierten oder sozial isolierten Personen könnte der Fall eintreten, dass nicht innert kurzer Frist eine Wohnmöglichkeit sowie Arbeitsmöglichkeiten zur Verfügung gestellt werden könnten, womit die Reintegration durch eine instabile Entlassungssituation gefährdet wäre. Mangels Wohnmöglichkeiten wären sodann Personenansammlungen in den sozialen Auffangzentren möglich, was angesichts der bundesrätlichen Vorgaben, Personenkonzentrationen wann immer möglich tief zu halten, als fragwürdig erscheint. Ebenso könnte sich bei Inhaftierten mit Landesverweis je nach Sachlage die Situation einstellen, dass sie von der einen in die andere Haft (Ausschaffungshaft) verlegt werden. In all diesen Fällen bliebe die mit der bedingten Entlassung angestrebte Risikoreduktion für die betroffene Person aus. 


\section{d) Alternative Lösungs- möglichkeiten}

32 Nach dem Gesagten ist klar, dass Art. 86 Abs. 4 StGB nicht das richtige juristische Modell ist, um den Infektionsrisiken im Freiheitsentzug zu begegnen. Unbeantwortet bleibt damit aber die Frage, wie zu verfahren wäre, wenn sich in kurzer Zeit mehrere inhaftierte Personen anstecken (Eskalationsszenario). Neben den einleitend erwähnten Massnahmen in Einrichtungen des Justizvollzugs selbst (vgl. I) wäre bei einer Infektion von inhaftierten Personen bei einer rapiden Verschlechterung des Gesundheitszustands der Weg nach Art. 80 StGB (Vollzug in einem Spital etc.) einzuschlagen. Der Kanton Zürich hat für die vorliegende Pandemie zusätzlich die Möglichkeit geschaffen, infizierte Personen einer spezialisierten Vollzugseinrichtung zuzuführen (Gefängnis Horgen).61 Damit könnte die Trennung von gesunden und angesteckten Personen erfolgen und für letztere eine adäquate Gesundheitsversorgung sichergestellt werden. Erst wenn trotz all diesen und den in der Einleitung geschilderten Präventionsmassnahmen (vgl. I) das Schutzniveau bei einer allfälligen unkontrollierten Verbreitung des Virus unzureichend wäre und der Staat seiner Schutzpflicht intramural nicht mehr nachkommen kann, wäre eine Unterbrechung des Vollzugs zu diskutieren (Art. 92 StGB). ${ }^{62}$

61 Medienmitteilung humanrights.ch (Fn. 12)

62 Zwar knüpft auch diese Norm an in der Person des Inhaftierten liegende Umstände an (BSKStGB-Koller, Art. $92 \mathrm{~N}$ 9). Jedoch könnte in einem Worst-Case-Szenario wie dem Geschilderten argumentiert werden, dass absolute Hafterstehungsunfähigkeit (bzw. Straferstehungsunfähigkeit) vorliegt, da bei einer solchen Sachlage den Gefahren für die Gesundheit der Inhaftierten nicht mehr intramural (auch nicht via Art. 80 StGB) begegnet werden kann. Vgl. auch Richtli-

\section{Abschliessende Bemerkungen}

33 Die COVID-19-Pandemie stellt den Justizvollzug vor grosse Herausforderungen und setzt ihn unter ständigen (gerechtfertigten) Legitimationsdruck. Auch in ausserordentlichen Lagen ist Art. 74 StGB der Leitstern sämtlicher vollzugsrechtlicher Überlegungen. Die Rechte von inhaftierten Personen dürfen «nur so weit beschränkt werden, als der Freiheitsentzug und das Zusammenleben in der Vollzugseinrichtung es erfordern.» Dabei sind in einem dynamischen Umfeld mit erheblichen Unsicherheiten Entscheide zu treffen, um Leib und Leben von Inhaftierten und Mitarbeitenden zu schützen. In der Schweiz sind über 6500 Personen von strafprozessualem oder strafrechtlichem Freiheitsentzug betroffen. ${ }^{63}$ Würden für diese Personen nicht zur gebotenen Zeit die geeigneten, erforderlichen und zumutbaren Präventionsmassnahmen getroffen, könnte sich diese Unterlassung desaströs auswirken. Nimmt man die (fluktuierende) Mortalitätsrate des COVID-19 von ca. $2 \%$ als Anhaltspunkt, könnte eine flächendeckende Verbreitung im Vollzug bis zu 130 Tote nach sich ziehen. Liesse sich dieses Szenario auf unterlassene Präventionsmassnahmen im Justizvollzug zurückführen, wäre dies nicht nur eine menschliche Tragödie, sondern auch eine eklatante Verletzung der staatlichen Schutzpflicht. Präventionsmassnahmen sind daher unvermeidbar, um eine Verbreitung des Virus in Einrichtungen des Freiheitsentzugs zu verhindern.

nie der Konkordatskonferenz des Strafvollzugskonkordats der Nordwest- und Inner-schweizer Kantone betreffend die Hafterstehungsfähigkeit vom 25. November 2016, $1 \mathrm{ff}$.

63 Zahlen des BFS über Freiheitsentzug, Insassenbestand nach Haftform. 
34 In der vorliegenden Abhandlung wurden zwei präventive Interventionsmöglichkeiten näher betrachtet, welche im Rahmen des Pandemieschutzes eine potenzielle Risikoreduktion herbeiführen könnten. Während die temporäre Einschränkung der Therapiefrequenz bei strafrechtlichen Massnahmen bei gleichzeitiger Gewährleistung einer therapeutischen Grundversorgung als verhältnismässiger Ausgleich zwischen zwei verfassungs- und konventionsrechtlichen Garantien eingestuft werden konnte (vgl. II.), musste die ausserordentliche bedingte Entlassung nach der Hälfte der Strafe (Art. 86 Abs. 4 StGB) als Lösungsmodell zurückgewiesen werden, weil sie erstens eine positive Prognose im Sinne von Art. 86 Abs. 1 StGB voraussetzt und die pandemiebegründeten Ansteckungsrisiken keine ausserordentlichen Umstände nach Art. 86 Abs. 4 StGB begründen können (vgl. III).

Die speziellen Rahmenbedingungen während der Pandemie - so auch die temporär eingeschränkten Therapieressourcen - bedeuteten für die inhaftierten Personen eine zusätzliche Bürde im Vollzugsalltag. Aus diesem Grund bleibt es die konstante Aufgabe des Justizvollzugs, den Zusatzlasten für die betroffenen Personen entgegenzuwirken und Nachteile im Rahmen der Verhältnismässigkeit auszugleichen. Abschliessend bleibt zu hoffen, dass aus der ausserordentlichen Lage nicht nur Belastungen, sondern mittel- und langfristig auch positive Folgen für die inhaftierten Personen resultieren. $\mathrm{Zu}$ nennen sind dabei insbesondere die erweiterten digitalen Kontaktmöglichkeiten für Inhaftierte. Nutzt man das bestehende Momentum auch nach Ende der Pandemie, könnte das unwillkommene Virus zu einer sehr will- kommenen Weiterentwicklung der Vollzugspraxis in das digitale Zeitalter beitragen. 